\title{
高效圆偏振发光的两种新策略：手性发光分子界面自组装和电荷转移 态发光
}

韩布兴

中国科学院化学研究所, 北京 100190

\section{New Approaches to Enhance Circularly Polarized Luminescence: Interfacial Self-Assembly of Chiral Emitters and Emissive Charge-Transfer Complexes}

\section{HAN Buxing}

Institute of Chemistry, Chinese Academy of Sciences, Beijing 100190, P. R. China.

Email: hanbx@iccas.ac.cn.

Published online: April 28, 2019.
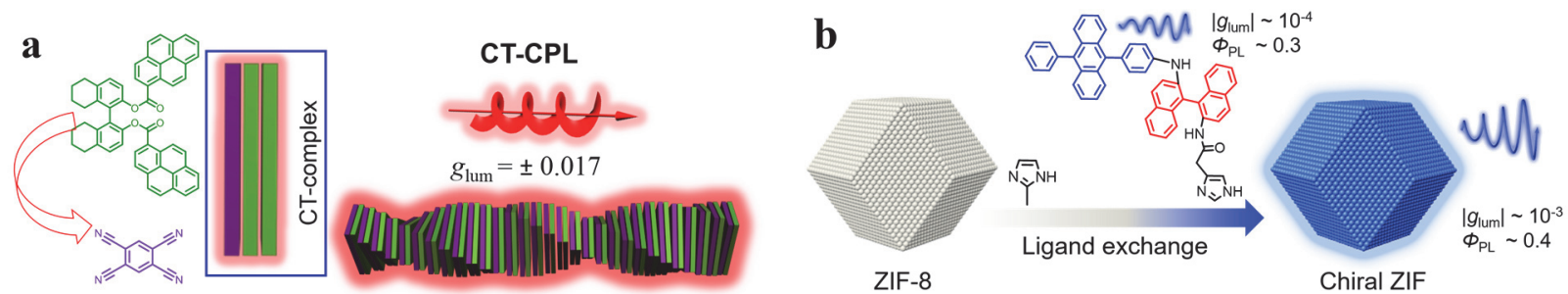

增强圆偏振发光的新方法: (a)电荷转移复合物; (b)手性发光分子界面自组装同时增强发光不对称因子及发光效率。

发光不对称因子 $\left(g_{\mathrm{lum}}\right)$ 和发光量子效率 $\left(\Phi_{\mathrm{PL}}\right)$ 是评估圆偏振发光(CPL)材料性能的两个重要的

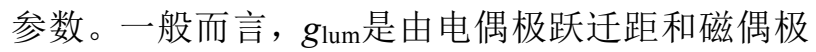
跃迁距决定的。有机体系中, 相比于电偶极跃迁 距, 磁偶极跃迁距往往是可以忽略的 1,2 。因此, 具 有较大电偶极的有机小分子其苂光量子效率很 高, 但是其不对称因子往往很小, 即 $g_{\mathrm{lum}}$ 和 $\Phi_{\mathrm{PL}}$ 是存 在一个此消彼长的矛盾关系。在一定程度上来讲

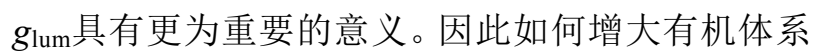
圆偏振发光的不对称因子 ${ }^{3-7}$, 更进一步如何构筑 兼具高发光不对称因子和高发光效率的有机材料 依然是该研究领域的关键性问题。

最近, 国家纳米科学中心段鹏飞研究员课题 组和中国科学院化学研究所刘鸣华研究员课题组 合作, 制备了具有发光性能的手性电荷转移 (charge transfer, CT)复合物材料, 该报道首次实现
了电荷转移复合物的圆偏振发光, 并且利用电荷 转移复合物具有顺磁性的性质, 得到了较大的圆 偏振发光不对称因子; 同时他们还制备了一种具 有手性发光性质的沸石咪唑型金属有机框架 (MOFs)材料, 利用手性发光分子在MOF材料表面 的有序自组装, 实现了圆偏振发光不对称性和荧 光量子效率的同时放大。相关工作发表在 Angewandte Chemie International Edition 上, $^{8}$ 。

他们合成了含有萠环的手性发光分子作为电 子供体。研究发现手性的电子给体可以与多种电 子受体形成复合物, 由于部分电子受体的最低未 占分子轨道(LUMO)能级较深, 形成的复合物并不 发光。具有合适能级的四氰基苯(TCNB)与电子给 体不仅可以形成手性 $\mathrm{CT}$ 复合物, 而且还具有 $\mathrm{CT}$ 态

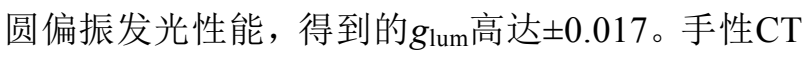
复合物可以通过多种方法得到, 包括共结晶、共研 
磨、旋涂等。更有趣的是电子给受体溶液混合之后 在超声的作用下可以形成 CT复合物的超分子凝 胶。在缺少像氢键等较强分子间非共价相互作用 的情况下, 仅通过 CT作用就可以形成超分子凝胶 还是很少见的。同时超分子凝胶也表现出较大的 圆偏振发光不对称因子。

为了得到兼具高发光不对称因子和发光量子 效率的有机体系, 他们选择了一种常见的沸石咪 唑型框架材料ZIF-8作为模板, 由于其优异的结晶 性能, 非常容易获得尺寸和结构可控的菱形十二 面体纳米材料。将具有圆偏振发光性能的手性有 机分子通过配体交换, 重组到ZIF-8的表面, 从而 获得具有圆偏振发光性能的ZIF材料。通过该方法 获得的手性ZIF的 CPL 发光不对称因子 $g_{\text {lum }}$ 相比于 手性分子本身提高了一个数量级。同时其发光量 子效率也有明显的提高。通过红外光谱、粉末 X射 线衍射和原子力显微镜等手段表征了手性分子在 ZIF表面的自组装状态, 发现其表现出规则的组装 性质。因此手性分子在ZIF表面良好规整的排列是 圆偏振发光不对称因子放大的根本原因。同时, 发 光分子与 $\mathrm{Zn}$ 离子配位之后分子构象的锁定可以降 低激发态分子的非辐射跃迁, 因此其发光效率得 到提高。

这些工作为提升有机体系发光不对称因子及
构筑高发光不对称因子和发光量子效率的有机材 料开辟了新的思路和方法。

\section{References}

(1) Sánchez-Crnerero, E. M.; Agarrabeitia, A. R.; Moreno, F.; Maroto, B. L.; Muller, G.; Ortiz, M. J.; de la Moya, S. Chem. Eur. J. 2015, 21, 13488. doi: 10.1002/chem. 201501178

(2) Kumar, J.; Nakashima, T.; Kawai, T. J. Phys. Chem. Lett. 2015, 6, 3445. doi: 10.1021/acs.jpclett.5b01452

(3) Liu, J.; Su, H.; Meng, L.; Zhao, Y.; Deng, C.; Ng, J. C. Y.; Lu, P.; Faisal, M.; Lam, J. W. Y.; Huang, X.; et al. Chem. Sci. 2012, 3, 2737. doi: $10.1039 / \mathrm{C} 2 \mathrm{SC} 20382 \mathrm{~K}$

(4) Ji, L.; Sang, Y.; Ouyang, G.; Yang, D.; Duan, P.; Jiang, Y.; Liu, M. Angew. Chem. Int. Ed. 2019, 58, 844. doi:10.1002/anie.201812642

(5) Han, J.; Duan, P.; Li, X.; Liu, M. J. Am. Chem. Soc. 2017, 139, 9783. doi: $10.1021 /$ jacs.7b04611

(6) Yang, D.; Duan, P.; Zhang, L.; Liu, M. Nat. Commun. 2017, 8, 15727. doi: $10.1038 /$ ncomms 15727

(7) Kumar, J.; Nakashima, T.; Tsumatori, H.; Kawai, T. J. Phys. Chem. Lett. 2014, 5, 316. doi: 10.1021/jz402615n

(8) Zhao, T.; Han, J.; Jin, X.; Liu, Y.; Liu, M.; Duan, P. Angew. Chem. Int. Ed. 2019, 58, 4978. doi: 10.1002/anie.201900052

(9) Han, J.; Yang, D.; Jin, X.; Jiang, Y.; Liu, M.; Duan, P. Angew. Chem. Int. Ed. 2019, doi: 10.1002/anie. 201902090 